\title{
A Polarization-Imaging-Based Machine Learning Framework for Quantitative Pathological Diagnosis of Cervical Precancerous Lesions
}

\author{
Yang Dong, Jiachen Wan, Xingjian Wang, Jing-Hao Xue, Jibin Zou, Honghui He, Pengcheng Li, Anli \\ $\mathrm{Hou}$, and Hui Ma
}

\begin{abstract}
Polarization images encode high resolution microstructural information even at low resolution. We propose a framework combining polarization imaging and traditional microscopy imaging, constructing a dualmodality machine learning framework that is not only accurate but also generalizable and interpretable. We demonstrate the viability of our proposed framework using the cervical intraepithelial neoplasia grading task, providing a polarimetry feature parameter to quantitatively characterize microstructural variations with lesion progression in hematoxylin-eosin-stained pathological sections of cervical precancerous tissues. By taking advantages of polarization imaging techniques and machine learning methods, the model enables interpretable and quantitative diagnosis of cervical precancerous lesion cases with improved sensitivity and accuracy in a lowresolution and wide-field system. The proposed framework applies routine image-analysis technology to identify the macro-structure and segment the target region in H\&Estained pathological images, and then employs emerging polarization method to extract the micro-structure information of the target region, which intends to expand the boundary of the current image-heavy digital pathology, bringing new possibilities for quantitative medical diagnosis.
\end{abstract}

Index Terms-Cervical precancerous tissues, Dualmodality machine learning, Polarization imaging, Quantitative pathological diagnosis.

\section{INTRODUCTION}

$\mathrm{T}$ HE process of digitizing the whole slide of tissue section has led to the advent of machine learning (ML) tools in computational digital pathology, which have been applied to various tasks, including disease diagnosis and prognosis of treatment based on patterns in pathological images [1]. Several ML applications in digital pathology have focused on the need to automate the time-consuming tasks for pathologists, subsequently enabling them to pay more attention on high-level decision-making tasks [2]-[4]. Cervical cancer, as a disease affecting more than half a million women every year, is the fourth most common woman malignancy worldwide [5], and most cases occur in underdeveloped areas where medical resources are limited [6]. It may take many years for cervical cancer to progress from pre-malignancy to invasion or metastasis, and most women with early-stage tumors can be cured by effective therapies [7]. Therefore, by the introduction of effective diagnosis of cervical precancerous lesions, the incidence and mortality of cervical cancer can be significantly reduced, and the validity of treatments and prognoses can be greatly improved [8]. Clinically, the gold standard of diagnosis is based on the histology evaluation of microscopic images by experienced pathologists, enabling the severity of cervical precancer to be determined [9]. Cervical precancerous lesions, known as cervical intraepithelial neoplasia (CIN), are classified as CIN1 (mild dysplasia), CIN2 (moderate dysplasia), or CIN3 (severe dysplasia) according to the increasing level of immature atypical cells across the epithelium region [10], [11]. During the diagnosis process, it is difficult to achieve accurate CIN classification via only observing and evaluating the hematoxylin-eosin (H\&E) stained pathological sections of cervical tissues, especially for interns. Therefore, the immunohistochemical (IHC) examination is required to accurately evaluate the lesion severity at the molecular level for

This work was supported in part by National Natural Science Foundation of China (NSFC) (Grant Nos. 61527826 and 11974206 ), Shenzhen Bureau of Science and Innovation (Grant No. JCYJ20170412170814624), and Shenzhen Guangming District Bureau of Science and Innovation (Grant No. 2020R01043). (These authors contributed equally: Yang Dong; Jiachen Wan. Corresponding authors: Anli Hou; Hui Ma.)

Y. Dong, X. Wang, P. Li, and H. Ma are with Center for Precision Medicine and Healthcare, Tsinghua-Berkeley Shenzhen Institute, Tsinghua University, Shenzhen, 518071, China (e-mail: mahui@tsinghua.edu.cn).

J. Wan is with Department of Applied Physics, New York University, Tandon School of Engineering, Brooklyn, NY11201, USA.

J.-H. Xue is with Department of Statistical Science, University College London, London, WC1E 6BT, UK.

J. Zou is with Department of Pathology, University of Chinese Academy of Sciences Shenzhen Hospital, Shenzhen, 518106, China.

$\mathrm{H}$. He and $\mathrm{H}$. Ma are with Guangdong Engineering Center of Polarization Imaging and Sensing Technology, Shenzhen Key Laboratory for Minimal Invasive Medical Technologies, Tsinghua Shenzhen International Graduate School, Tsinghua University, Shenzhen, 518055, China.

A. Hou is with Department of Gynecology, University of Chinese Academy of Sciences Shenzhen Hospital, Shenzhen, 518106, China (e-mail: doctorh2008@163.com)

H. Ma is with Department of Physics, Tsinghua University, Beijing, 100084, China. 
the cases that are difficult to diagnose [12], which is timeconsuming and laborious for pathologists and financial costly for patients. In addition, stroma and background, occupying the majority of the microscopic image area, are not the regions of interest during the analysis, making the manual examination of the whole slide image and identification of the epithelium region a tedious job. The above rationales suggest the necessity of automated segmentation of epithelium regions and quantitative diagnosis of cervical precancerous lesions in H\&Estained pathological images.

There is a clear pipeline in the existing image-based CIN diagnosis models: first the epithelium region is identified and extracted, followed by a texture-feature based or deep-learning based model attempting to make a classification decision according to the image features of epithelium region. De et al. used texture features for the analysis of the epithelium region images, achieving a classification accuracy of $88.5 \%$ by using the linear discriminant analysis classifier when classifying Normal versus CIN samples [13]. Wang et al. used texture features and statistical moments combined with a support vector machine (SVM) model to segment epithelium regions with the accuracy of $94.2 \%$, defined 'perpendicular line features' based on nuclei features, and achieved an accuracy over $95.0 \%$ on the classification of Normal vs CIN [14]. Sornapudi et al. proposed a convolutional neural network (CNN) based framework for CIN classification, consisting of a ResNet based model to segment out epithelium regions with the Jaccard index of $94.0 \%$ [15], and a two-fold network named DeepCIN to perform final classification based on the segmented epithelium. An accuracy of $92.6 \%$ for the classification of Normal vs CIN was achieved [16]. Xu et al. used super-pixels combined with deep convolutional neural networks (DCNN) to segment epithelium regions, with an accuracy of $88.0 \%$ on H\&E-stained images [17]. For the segmentation of epithelium in histological images, U-net architecture [18] is used either as the main model [19], [20] to be optimized or as the benchmark model for comparison [15]. In this paper, a U-net architecture was used for epithelium segmentation and laid foundations for deriving CIN diagnosis indicators based on the sample's polarization features.

Mueller matrix contains abundant optical properties and microstructural information of complex biological specimens and provides label-free and comprehensive descriptions on the polarization properties of tissues [21]. Mueller matrix imaging has been applied in various biomedical applications, including characterization of target microstructures for both backward scattering imaging of bulk tissue samples [22] and transmission imaging of thin tissue slices [23]. For example, for bulk tissues: Schucht et al. [24] employed backscattering wide-field imaging Mueller polarimetry for visualization of white matter fiber tracts of brain tissue, which could improve the accuracy of tumor border detection and patient outcome prediction; For in vivo imaging and even in situ examinations, Qi et al. [25] designed a high-definition Mueller polarimetric endoscope for tissue characterization. This technique has potential applications in surgical margin detection, wide field early epithelial cancer diagnosis, and energy-based tissue fusion monitoring; Dong et al. [26] proposed a quantitative, noncontact technique to monitor the microstructural variations of skin tissues during ultraviolet photo-damage based on Mueller matrix imaging. In addition, for the detection of thin tissue samples, we have developed transmission Mueller matrix microscopes with the advantages of simple structure, fast imaging speed, and low cost, and examined the pathological samples' Mueller matrix images together with the pathological color images, which shows good practicable prospects in the preliminary clinical diagnosis. To disentangle the information encoded in the 16 Mueller matrix elements, sets of parameters with physical meanings have been derived from Mueller matrices by different approaches [27]-[30]. These polarization parameters demonstrated promising potentials for characterizing microstructural features in various pathological tissues [28]. Previously, we used the polarization parameters for the diagnosis of breast cancer [31], Crohn's disease [32], liver fibrosis [33], [34], and so on. Furthermore, we proposed a linear discriminant analysis (LDA) based approach for deriving polarization parameters used for quantitative characterization of cell nuclei and fiber collagen in various breast pathological tissues [35]. Previous experiments and simulations have shown that polarization properties of complex samples are sensitive to microstructure down to sub-wavelength scales, which scatters polarized light and alters its polarization. Therefore, whereas ordinary images rely on spatial resolution to reveal detailed texture information, polarization images encode subwavelength microstructural features even at low-resolution and wide-field conditions [36]-[38].

In this study, we proposed a polarization-imaging-based ML framework combining polarization imaging and traditional microscopy imaging, and thus constructed a dual-modality machine learning framework for quantitative diagnosis of cervical precancerous lesions, as shown in Fig. 1. Firstly, we took microscopic Mueller matrix images and H\&E images of the whole slides of different cervical pathological tissues Normal, CIN1, CIN2, and CIN3 under a $2.5 \times$ objective. Pixel level registration was achieved between sample's Mueller matrix image and its $\mathrm{H} \& \mathrm{E}$ image using affine transformation. Then sets of polarization parameters in the regions of interest (ROI) from earlier studies [27]-[30] were calculated from Mueller matrix images as the polarimetry basis parameters (PBPs) (Table $\mathrm{SI}^{1}$ ). In the H\&E images of cervical tissue samples, the epithelium regions were segmented by using a Unet, and a mask was generated to select the pixels of epithelium regions in PBPs, which were used as input data for the designed statistical distance-based ML classifier for deriving a polarimetry feature parameter (PFP). PFP is a simplified linear function of the PBPs with physical meanings, and the statistical distribution of PFP may closely relate to the specific microstructural variations as cervical lesion progresses. We demonstrated the feasibility of this polarization-imaging-based ML framework by evaluating the performance of the U-net and

\footnotetext{
${ }^{1}$ Table SI is available in Section II of the Supplementary document.
} 


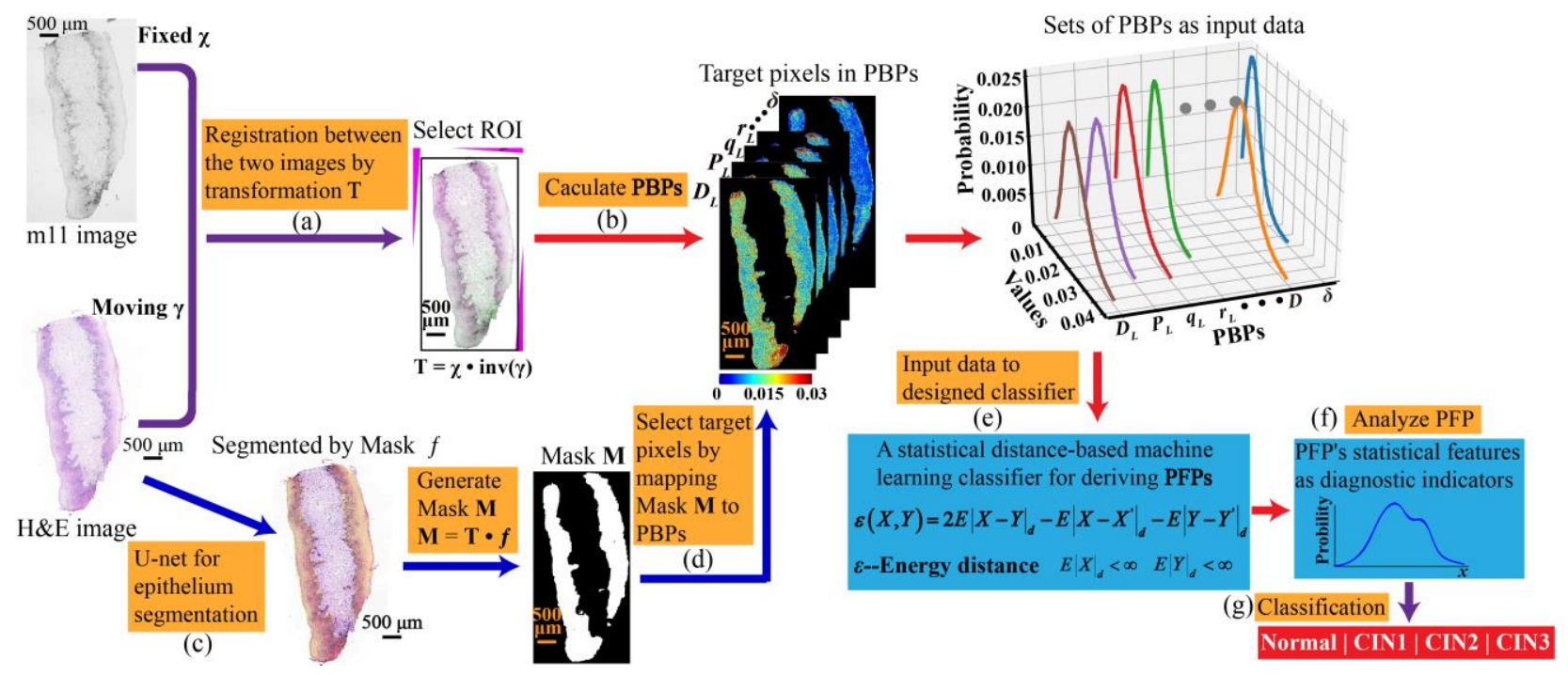

Fig. 1. Polarization-imaging-based ML framework for quantitative diagnosis of cervical precancerous lesions. The schematic illustrates a fusion method of dual-modality information contained in the tissue sample's Mueller matrix and H\&E image, and (a)-(g) outlines the steps from the input of Mueller matrix image and H\&E image of the cervical tissue sample to the output of PFP for obtaining CIN diagnostic indicators.

PFP. The experimental results demonstrated that the U-net can generate a reliable mask to select target pixels in PBPs images and the statistical parameters of the derived PFP could be used as potential indicators when classifying the cervical pathological samples, which is expected to automate the diagnosis of cervical precancerous lesions and reduce the workload of pathologists. The proposed framework applies routine image-analysis technology to identify the macrostructure and segment the target region in H\&E images, and then employs emerging polarization method to extract the micro-structure information of the target region. Through the complementarity between the H\&E-stained pathological image and the polarization information of tissue samples, our technique has profound significance for accurate and quantitative digital pathology diagnosis.

\section{METHODS}

\section{A. Cervical Pathological Samples and Experimental Setup}

The H\&E-stained pathological slides of cervical tissues used in this study (4- $\mu$ m-thick) were acquired from cases in the University of Chinese Academy of Sciences Shenzhen Hospital. 140 pathological samples from a total of 140 patients diagnosed as Normal, CIN1, CIN2, and CIN3 were analyzed (45 cases for Normal, 45 cases for CIN1, 25 cases for CIN2, and 25 cases for CIN3). Fig. 2 (a) shows the pathological features in epithelium region (marked by blue solid line) of different cervical tissue specimens: as the severity of the dysplasia increases, the density of immature atypical cells increases from the basal layer (indicated by red arrows) to the superficial layer (indicated by black arrows) of the epithelium. Here, pathologists labelled the epithelium regions in H\&E images of cervical tissues manually using MATLAB Graphical User Interfaces (GUIs). The GUI reads the H\&E image, shows it to expert pathologists to label the epithelial area manually, and generates a binary image of the epithelial area as the ground truth for the training and testing of U-net. The GUI calls the imfreehand function to draw the ROI freely and manually on the image without using other image analysis methods. Besides, in clinic, pathologists have a variety of diagnostic methods of CIN samples, for example, observing and evaluating the severity of microstructural changes in $\mathrm{H} \& \mathrm{E}$ images, using immunohistochemistry examination to evaluate the lesion severity at the molecular level, and so on. The classification label of each cervical sample needs to be provided by pathologists based on these clinical methods as the ground truth for the training and testing of ML classifier. This work was approved by the Ethics Committee of University of Chinese Academy of Sciences Shenzhen Hospital.

The dataset for this research consisted of cervical pathological samples' H\&E images and Mueller matrix images, which were captured by using the Mueller matrix microscope, as shown in Fig. 2 (b). By adding two compact modulespolarization state generator and analyzer (PSG and PSA) to the existing optical path of the transmission-light microscope, the experimental setup can measure sample's Mueller matrix based on the dual rotating retarder method [33], [39]. During measurement, different polarization states are implemented by two fixed linear polarizers P1, P2 and two rotating quarter-wave retarders R1, R2, and 30 intensity images with different polarization states are collected by the grayscale CCD. And thus Mueller matrix image can be calculated from the 30 intensity images. Before being applied to tissue samples, we calibrated the microscope by measuring the Mueller matrices of standard samples, such as air, and the experimental results testified that the maximum error of the Mueller matrix microscope is about $1 \%$. The details about the Mueller matrix measurement and calibration are provided in Section I of the 
Supplementary document ${ }^{2}$. Moreover, in the microscope system, we can also obtain the histological images of tissue samples from color CCD by turning off the optical path where photons enter the grayscale CCD.

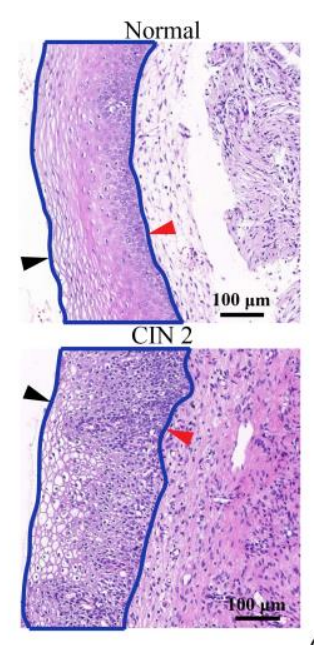

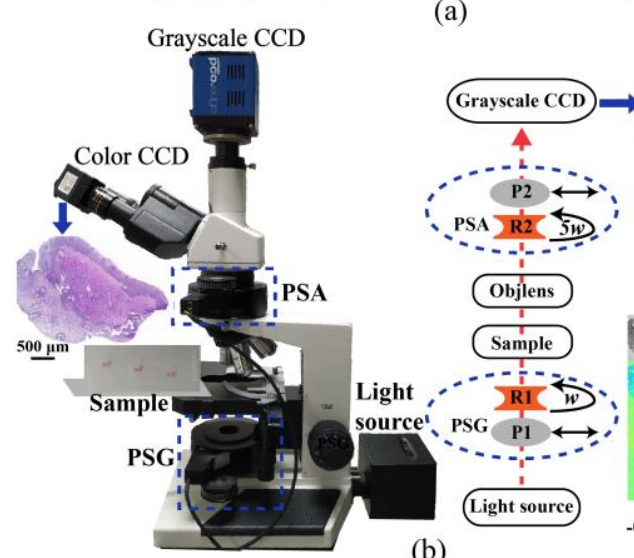

(b)

(a)
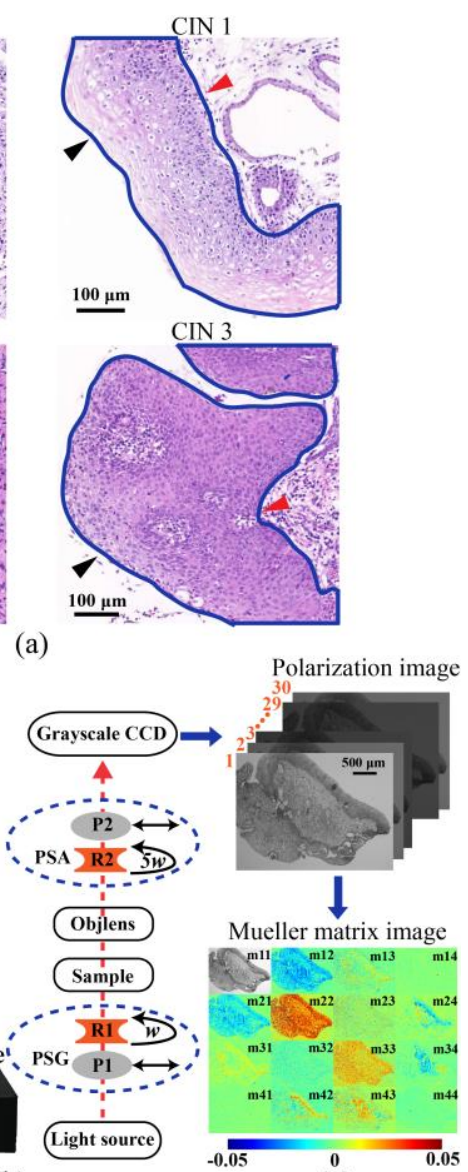

(c)
Fig. 2. Cervical pathological samples and experimental setup. (a) Example H\&E images of different cervical pathological tissues - Normal, CIN1, CIN2, and CIN3. (b) Photograph and schematic of the Mueller matrix microscope. P: polarizer. R: quarter-wave plate. (c) An example of Mueller matrix of the pathological section of CIN2. The element m11 represents the intensity image, and the other 15 elements are all normalized by $\mathrm{m} 11$. We subtract the identity matrix from the Mueller matrix for display using the color bar ranging from -0.05 to 0.05 .

\section{B. Polarimetry Basis Parameters}

Mueller matrix elements contain complete polarization properties of samples. However, individual Mueller matrix elements lack explicit connections to the sample's microstructural characteristics, and are often seriously affected by sample orientations [40], [41]. Recently, multiple techniques were adopted to derive several sets of PBPs which have clear physical meanings and are either insensitive or related explicitly to the sample's orientation angle [27]-[30]. Table SI ${ }^{3}$ summarizes the computing formulas and physical meanings of PBPs used in polarimetry. These parameters are all used as

\footnotetext{
2 More details about Mueller matrix measurement and calibration are available in Section I of the Supplementary document.

${ }^{3}$ Table SI is available in Section II of the Supplementary document.
}

input polarization features of samples in this study: Mueller matrix polar decomposition method was proposed by $\mathrm{Lu}$ and Chipman [27], deriving linear retardation $\delta$, diattenuation $D$, depolarization $\Delta$, and optical rotation $\psi$. Anisotropy degree $t_{1}$, polarizance $b$, circular birefringence $\beta$, and normalized anisotropy $A$ are from the Mueller matrix transformation method proposed in our previous study [28], [29]. Mueller matrix rotation invariant parameters - linear and circular polarizance $P_{L}$ and $P_{C}$, linear and circular diattenuation $D_{L}$ and $D_{C}$, linear birefringence related $r_{L}$ and $q_{L}$, and $k_{C}$ with different physical meanings in pure depolarization and linear retarder system - also decode effective information from the Mueller matrix [30]. To fully mine the information encoded in the Mueller matrix of samples, here we proposed series of Mueller matrix linear birefringence identity parameters $\left(P_{1}, P_{2}, P_{3}\right.$, and $\left.P_{4}\right)$ and linear diattenuation identity parameters $\left(P_{5}, P_{6}, P_{7}\right.$, and $\left.P_{8}\right)^{4}$ based on the Mueller matrices of linear retardance and linear diattenuation respectively [42]. These parameters can be used as indicators for deviation of an asymmetric anisotropic Mueller matrix compared with a pure linear retarder Mueller matrix or a pure linear diattenuator Mueller matrix.

\section{Data Preprocessing}

In this study, the input data of the statistical distance-based ML classifier for deriving a PFP was the target pixel values in PBPs. These pixels were selected by the epithelium region segmentation maps in the $\mathrm{H} \& \mathrm{E}$ images of the cervical pathological tissues. The automatic segmentation of epithelial regions in H\&E images was performed by the trained U-net model and produced the epithelium region mask for directly mapping on the samples' PBPs images to select pixels. Therefore, we need to achieve pixel by pixel registration between sample's Mueller matrix image and H\&E image before selecting pixels by direct mapping. The element $\mathrm{m} 11$ in Mueller matrix represents the intensity image of the sample, which can be used as the fixed image for pixel level registration with the moving image - the H\&E image of the sample. As shown in Fig. 1 (a), we adopted the affine transformation method [43], [44] to transform the H\&E image to match the $\mathrm{m} 11$ image. In the affine transformation, the H\&E image was first transformed into grayscale image, which is used to register with $\mathrm{m} 11$, and then the transformation matrix $\mathbf{T}$ is calculated. Specifically, for rough registration, the optimizer and metric were configured using the imregconfig function in MATLAB (function parameter was set as multimodal). Then, by adjusting the parameters of the optimizer and optimizing the initial conditions, the accurate registration between the fixed image and the moving image was achieved using the imregister function in MATLAB. Considering translation, rotation, scale, and shear between the two images from the same tissue section, we set transform type in the imregister function as "affine". In the optimizer, the step size was reduced by 3.5 times and the

\footnotetext{
${ }^{4}$ Extraction method is available in Section II of the Supplementary document.
} 
maximum number of iterations was increased to 300 considering the tradeoff between time complexity and registration quality. After that, the transformation matrix $\mathbf{T}$ was calculated by the imregtform function. We calculated the PBPs of the overlapping region of the two images and transformed the epithelium region mask using $\mathbf{T}$ to select target pixels in PBPs as input data of the classifier.

\section{Algorithm Architecture}

The Polarization-imaging-based ML framework consists of a modified U-net model for epithelial region segmentation, a designed statistical distance-based ML classifier for deriving a PFP, a PFP analyzing system for illustrating the polarization features of target microstructures in cervical precancerous lesions, and a CIN classifier for final CIN classification, as shown in Fig. 1. This framework combines the dual-modality image information for quantitative diagnosis of cervical precancerous lesions, where architecture and parameters are illustrated as follows.

1) U-net

As shown in Fig. 3, the modified U-net model has only 3 levels in depth, which consists of a contracting path which follows the typical architecture of a convolutional network, and an expansive path by up-sampling of the feature map [18]. During the down-sampling path, in each level, the network applies two $3 \times 3$ convolution layers with ReLU activation and a $2 \times 2$ Max pooling operation. During the up-sampling path, the architecture is mostly symmetric to the down-sampling path, only replacing the Max pooling operation with a $2 \times 2$ upconvolution. The last layer of the model is a $1 \times 1$ convolution layer with sigmoid activation. The filter sizes for the convolution operations in the three levels are set to 64,128 , and 256 , respectively.

The model was trained by using the Adam optimizer, with learning rate $0.0002, \beta \_10.9$, and $\beta \_20.99$. The learning rate is determined by grid search, based on the validation loss after 5 epochs with each learning rate setting. Loss functions, such as cross-entropy, Dice loss, and mean squared error, are tested, and mean squared error produced the most consistent result. The model allows to train for 100 epochs with early stopping. The model training and implementation were done using Python version 3.7.6 and Keras version 2.3.1.

\section{2) Statistical Distance-based ML Classifier}

In our ML model, we designed a loss function according to the requirements of cervical pathological diagnosis in clinical practice and data characteristics in polarimetry. Firstly, the main differences between cervical tissues with different severity of pathological changes lie in the proportion and distribution of immature atypical cells in the epithelial region. Fig. 2(a) also tells us that, it is a gradual process of lesionrelated microstructural variation from Normal to CIN3 samples. The previous experiments and simulations help us build such a prior knowledge - polarization parameters may act as quantitative indicators of microstructural gradual variations [26], [34]. For example, Wang et al. [34] demonstrated that as the severity of liver fibrosis increases, i.e., the fiber structures in liver pathological samples gradually increase, the values of birefringence-related parameters gradually increase. Therefore, as long as the PFP corresponding to lesion-related microstructural variation could be derived by the ML model, all the pathological stages of cervical lesions are expected to be quantitatively distinguished based on the gradual change and accumulation of the samples' polarization features. Inspired by this, we only used the target pixels in PBPs images of Normal cervical tissue samples labelled as negative class and those of CIN1 samples labelled as positive class to train the model for deriving such a PFP, which highlights the advantages of dual-modality framework from fully utilizing polarization imaging technique. In addition, it is expected that PBPs' characteristics inside the epithelial regions of normal samples is marginally different from that of the CIN1 samples, given that most microstructures inside the epithelial regions of CIN1 samples are similar to Normal. We thus look for a distributional difference between the two sets. Therefore, the loss function is designed based on the statistical distance between polarization features distribution of different classes:

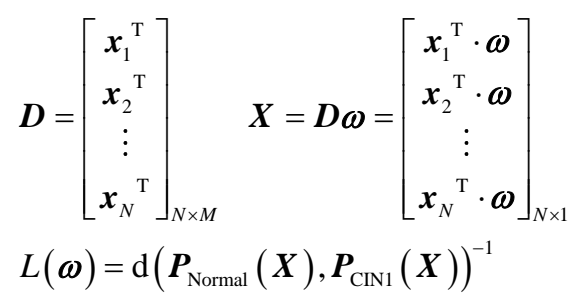

where $\boldsymbol{x}_{i}$ is a $M \times 1$ vector, $i \in[1,2, \ldots, N]$, and represents the PBPs values of a pixel in the epithelial region. $M$ and $N$ are the number of PBPs and the number of target pixels respectively. Thus, $\boldsymbol{D}$ is a $N \times M$ data matrix. $\boldsymbol{\omega}$ is a $M \times 1$ vector, representing weight coefficients of PBPs. $\boldsymbol{X}$ is a $N \times 1$ vector calculated as the linear projection of the input PBPs. $\boldsymbol{P}_{\text {Normal }}(\boldsymbol{X})$ is the probability distribution of $\boldsymbol{X}$ from Normal cervical pathological tissues, and $\boldsymbol{P}_{\mathrm{CIN} 1}(\boldsymbol{X})$ is the probability distribution of $\boldsymbol{X}$ from CIN1 tissues. $\mathrm{d}$ is the energy distance [45] between $\boldsymbol{P}_{\mathrm{Normal}}(\boldsymbol{X})$ and $\boldsymbol{P}_{\mathrm{CIN} 1}(\boldsymbol{X})$. The statistical distance-based training algorithm learns to minimize the loss function by gradient descent (initial learning rate 1 , learning rate decay 0.99 , tolerance 0.01 ), which means to maximize the distance between polarimetry feature distributions of Normal cervical tissues and those of CIN1 tissues. Then the features with coefficient close to 0 are filtered out to reduce the number of PBPs used for deriving the PFP.

3) PFP Analyzing System

After deriving the PFP, we adopted a PFP analyzing system to demonstrate the correlation between the changes of polarization characteristics and the pathological development of cervical precancerous lesions, which may bring physical interpretability (presented in Results. B.) for the pathological mechanism (presented in Fig. 2(a)). Here, the basic statistical 


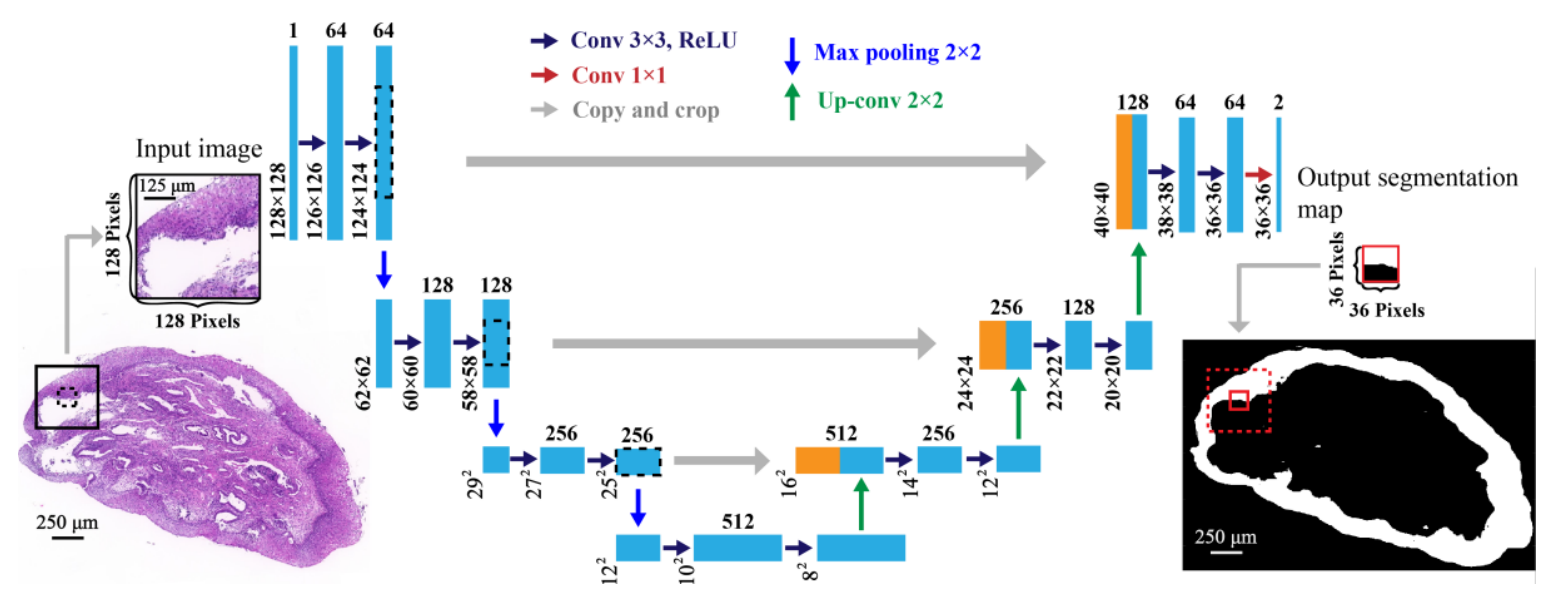

Fig. 3. Architecture of U-net for epithelial segmentation in cervical H\&E images. Blue boxes correspond to multi-channel feature maps, and orange boxes represent copied feature maps. It consists of a contracting path and an expansive path (left side and right side respectively). This model has 3 levels in depth, and the filter sizes for the convolution layers in the 3 levels are 64, 128, and 256 respectively. The x-y size of the image is displayed at the lower left edge of the boxes. The arrows denote the different operations. A segmentation example in H\&E image of a CIN2 pathological sample is presented.

characteristics (shown in Table SII ${ }^{5}$ ) [46] of PFP's probability distribution were calculated, including mean, standard deviation, mode, skewness, kurtosis, energy, entropy, and full width at half maximum. To show the relationship more intuitively between PFP's statistics and lesion-related microstructural changes, we traverse all possible combinations of three statistical features of PFP and select three typical statistical features with the highest accuracy of classification of Normal vs CIN. Specifically, the training samples contain 20 Normal cervical tissue samples and 20 CIN1 samples, and their PFP's statistical features were traversed, and three features were input into a linear binary SVM classifier [47] for training. We fed the corresponding three PFP's statistical features of 15 normal tissues and 15 abnormal tissues in the validation samples into the trained SVM classifier and calculated the classification accuracy. We selected the three statistical features as typical features when the classification accuracy achieved the highest.

To further reduce the dimension and explain the relationship between the target microstructural variation at different pathological stages and the change trends of PFP's statistical characteristics, we calculated the line of best fit using the first component of principal component analysis (PCA) [48]. Here, we adopted singular value decomposition (SVD) to perform PCA, and then the three-dimensional statistical features can be reduced to one-dimensional features, which can more conveniently summarize the process of microstructural variations.

\section{4) CIN Classifier}

For better clinical application, we used all of the PFP's statistical parameters to train a four-class SVM classifier to directly predict the four pathological stages, and a binary-class SVM for screening of abnormal samples from normal samples. There are 60 cases diagnosed as different cervix conditions (15 cases from each cervix condition) were used for the training of
CIN classifier. We used 5-fold cross validation method to determine the hyper-parameters in a four-class SVM and in a binary-class SVM classifier. In each pathological stage, three cases were randomly divided into a group. There were five groups in each stage which were taken turns as the test samples, meanwhile the rest were treated as the training samples. By this, we could obtain an optimized four-class SVM classifier and an optimized binary-class SVM classifier.

These SVM classifiers were implemented through the opensource library Scikit-learn in Python version 3.7.6, with class SVC. The linear kernel was used, with a regularization coefficient $C$ chosen as 12.6 and 100.7 respectively in the fourclass SVM classifier and the binary-class SVM classifier through 5-fold cross validation.

\section{RESULtS}

\section{A. U-net Performance for Epithelial Segmentation}

For segmentation, we used a shrunk version of the original U-net model with only 3 levels deep [18]. When an input H\&E image with size $128 \times 128 \times 3$ is fed to the designed model, the model produces a prediction with size $36 \times 36 \times 2$ for epithelial segmentation. To simplify the image registration process of H\&E images and polarization images, reduce the parameters size of U-net, and optimize the integration of the two modalities in the framework, the H\&E images have the same resolution as the polarization images, which are all obtained under a $2.5 \times$ objective lens. Details about the modified U-net architecture is shown in Fig. 3 and described in Methods. D. 1) section.

The dataset for the training and testing of U-net consisted of 140 H\&E-stained whole slides pathological images and corresponding manually segmented epithelial layer masks. In the dataset, 100 whole slides images representing the diversity of the data (35 images from Normal, 35 images from CIN1, 15 images from CIN2, and 15 images from CIN3) were employed

\footnotetext{
${ }^{5}$ Table SII is available in Section III of the Supplementary document.
} 
for training the model, and the remaining 40 whole slides images were used for testing and evaluating the model's performance on automatic segmentation of epithelial regions. During the training process, the images in the training set were cropped into $128 \times 128$ patches with stride 64 , and the 100 training images are augmented into 49870 image patches using data augmentation, including random cropping and random rotation, to increase model generalization. During the testing process, predictions were made with stride 36 to cover the entire test image. The model is trained using mean squared error as loss function with learning rate 0.0002 , determined empirically. The trained model achieved an overall pixel-wise accuracy of $94.4 \%$, and a Dice score of $90.1 \%$ for epithelial region segmentation in the test set.

\section{B. The Deriving of PFP}

To derive PFP for quantitative CIN classification, we randomly selected 20 Normal cervical tissue samples as negative class and 20 CIN1 samples as positive class among the 140 cases for training the statistical distance-based ML classifier. Fig. 4 (a) summarizes the architecture of the classifier. Details can be found in Methods. D. 2). The target pixels in PBPs were selected by projection from the segmentation map of H\&E image, which then served as input data for the designed classifier. The output PFP, as a simplest linear combination of PBPs (illustrated by Fig. 4 (b)), has physical interpretability: (1) complex cervical precancerous samples have polarization characteristics of various types of anisotropy superposition. The anisotropy produced by diattenuation plays an important role in characterizing the cervical pathology microstructural variations, resulting in larger coefficients of $D(18.4), D_{L}(19.3)$, and $P_{L}$ (22.6). The deviation between the sample's anisotropy and the PBPs sets of Normal samples

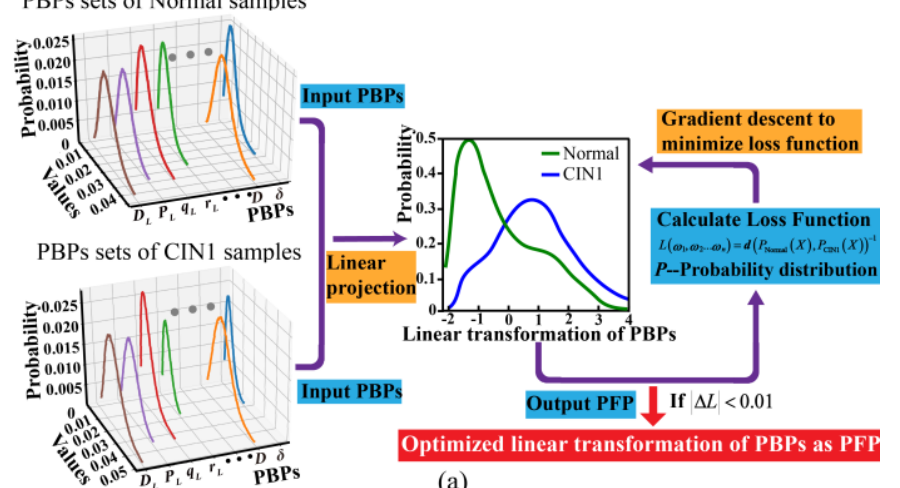

(a)

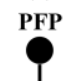

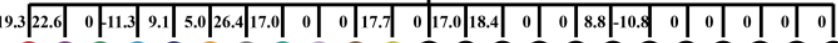

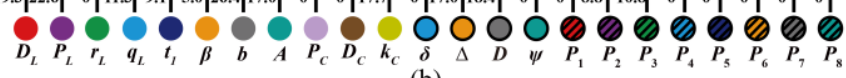

(b)

Fig. 4. Statistical distance-based ML classifier. (a) Schematic of the statistical distance-based ML classifier for deriving a PFP for the diagnosis of cervical precancerous lesions. (b) Linear combination of the PBPs (represented by solid circles in different colors and shapes) is output as the PFP (represented by solid circle in black). The numbers above PBPs are the optimized coefficients obtained from the trained classifier, and the PFP can be acquired by a weighted sum of the PBPs with optimized coefficients. pure linear retardance system accounts for a large proportion of the PFP composition, as shown in the coefficients of $P_{2}(8.8)$ and $P_{3}$ (10.8). The coefficient of $r_{L}$ is 0 , while that of $q_{L}$ is -11.3 , testifying birefringence asymmetry in sample's Mueller matrix and indicating that the anisotropy produced by birefringence also contributes much to the CIN grading. The overall anisotropy of cervical pathological samples can be reflected in the PBPs $t_{l}$ and $A$ whose coefficients are 9.1 and 17.0 respectively; (2) the PBPs $b$ (coefficient 26.4) and $\Delta$ (coefficient 17.0) are prominent in the function of PFP, which means that the depolarization ability of the cervical precancerous samples changes with the lesion development; (3) the PBP $k_{C}$ also contributes a lot to the quantitative characterization of microstructural changes, meaning that the polarized photons have the behavior changes of retardation and depolarization as they propagate and scatter in cervical pathological samples at different stages.

\section{Qualitative Results of the Polarization-imaging- based ML Model}

After obtaining the validation samples' H\&E images and sets of PBPs images using the Mueller matrix microscope, the performance of the polarization-imaging-based ML model can be validated. We feed the H\&E images of cervical pathological slides into the trained U-net for the segmentation of epithelium region. Then the PFP of epithelium region is calculated by the linear function presented in Fig. 4 (b). In Fig. 5, we summarize the segmentation results of a part of the epithelial region of the whole cervical pathological section and their corresponding 2D images of PFP. Fig. 5 (a) are parts of H\&E images of epithelial regions from cervical precancer tissues at different stages. For illustration, two examples (S1, S2) were taken from each pathological stage. Blue solid lines label the ground truth of epithelium regions in H\&E images by expert pathologists. Fig. 5 (b) are the segmentation results of epithelial regions in $\mathrm{H} \& \mathrm{E}$ images. These results show that the U-net can segment the epithelium region of cervical H\&E images, showing the correct region boundary that is expected from the pathologist determining ground truth in H\&E-stained tissue samples. In the segmentation results, white regions represent epithelial tissues, and black areas represent stromal tissue and background regions. Evaluation of Fig. 5 (b) shows that the boundary generated by the U-net is visually natural and less zigzag. Although the method produces erroneous boundaries as well, the errors appear to be subtle and not so egregious, which is acceptable to the pathologist.

Fig. 5 (c) shows 2D images of PFP from epithelium regions of cervical precancer pathological samples at different stages. In Fig. 5 (c), there is an obvious trend of increase in PFP with the development of cervical precancer lesions, indicating that the polarization characteristics represented by the PFP increase during the lesion progression. On the other hand, from Normal, CIN1 to CIN3 samples, the pixels with large values of PFP gradually spread from the basal layer (indicated by white arrows corresponding to the black arrows in Fig. 5 (a)) to the superficial layer of the epithelium tissue, resulting in the $2 \mathrm{D}$ patterns of PFP being gradually uniformly and discretely 


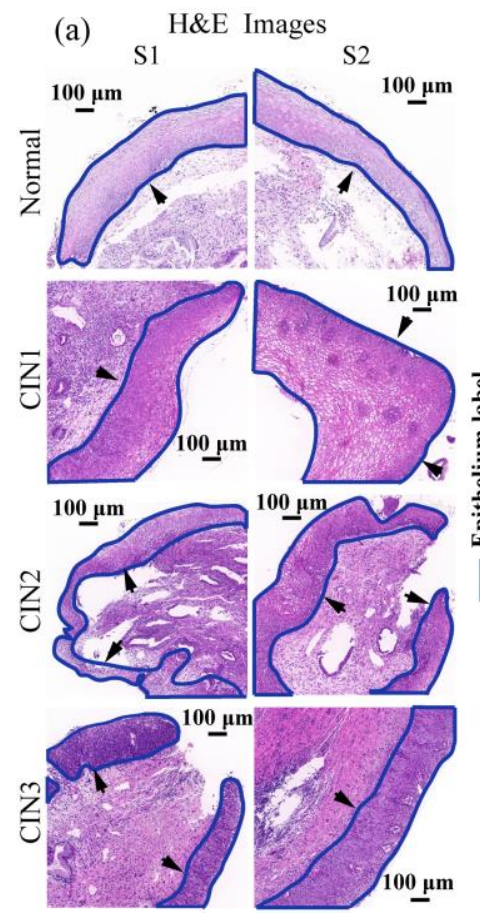

(b) Epithelium segmentation maps
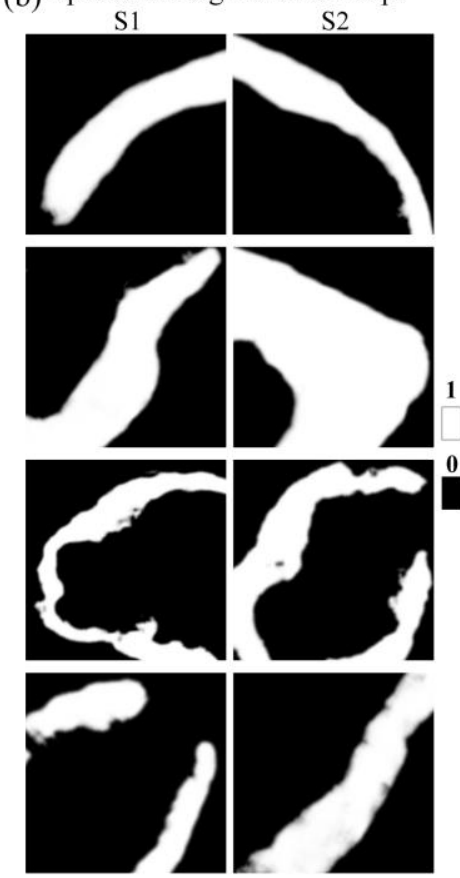

(c) PFP Images
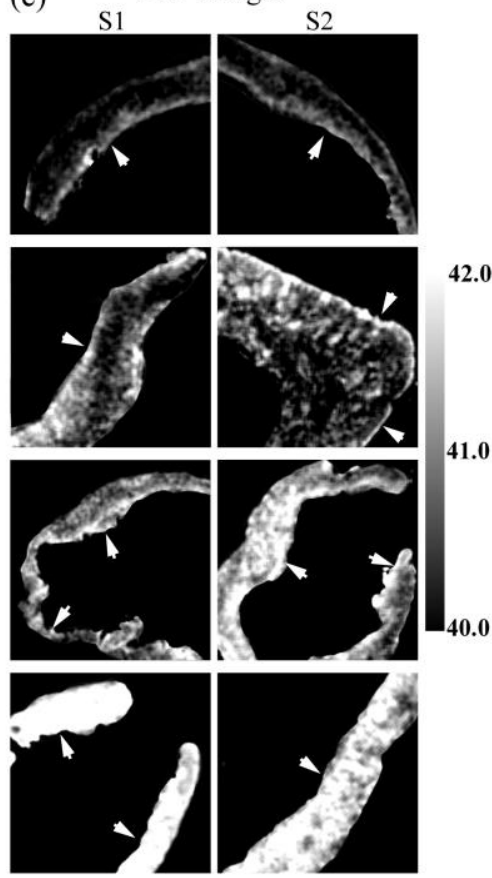

42.0

\section{.0}

Fig. 5. Qualitative results of the polarization-imaging-based ML model. S1, S2 represent two samples taken from cervical precancerous tissue at each pathological stage - Normal, CIN1, CIN2, and CIN3. (a) Parts of H\&E images of epithelial region of the whole cervical precancerous slides at different pathological stages. (b) Epithelium segmentation maps in the corresponding H\&E images. (c) 2D images of PFP of the corresponding epithelial regions.

distributed. In normal cervical pathological samples, there are few highlighted pixels, whereas the highlight pixels of PFP fill the whole epithelial regions in CIN3 samples. Therefore, from Normal to CIN3, the distributional behavior of PFP values (as shown in Fig. 5 (c)) is very similar to that of immature atypical cells (as shown in Fig. 5 (a)) across the epithelium region as the severity of the dysplasia increases, meaning there may be a close relationship between PFP's distribution and the lesion related microstructure variations.

D. Interpretable and Quantitative Relationship between PFP's Statistical Features and Pathological Variations

The PFP is expected to build connection with the pathological variations as cervical disease develops. This relationship is evaluated on the validation samples including 60 cases diagnosed as different cervix conditions (15 cases from each cervix condition). The validation samples are separated from the training samples which are used in the training of the statistical distance-based ML classifier for deriving PFP. The probability distributions of PFP of the 60 cervical samples are shown in Fig. 6 (a) from which we can observe that the positions, widths, and shapes of curves from the four cervical pathological stages are different. Specifically, the probability curve shifts to larger values of PFP as cervical lesion progresses and transforms from a positively-skewed distribution to a negatively-skewed distribution.

To quantitatively evaluate the PFP's distribution of different cervical pathological tissues, we conducted the PFP analyzing system (See Methods. D. 3)). According to the experiment results, the mean, mode, and skewness of PFP were selected as typical features for illustrating the relationship between the PFP and the microstructural variations as lesion progresses. Fig. 6 (b) reveals the values of the three typical features of the 60 validation samples, where $N$ is the line of best fit obtained by PCA. This line can be used for summarizing the features' variations in the three-dimensional coordinate system. We can conclude from Fig. 6 (b) that: (1) the probability distributions of PFP in most normal epithelial tissues are right-skewed (the skewness is larger than 0), and those in epithelial regions of CIN2 and CIN3 are left-skewed (the skewness is smaller than $0)$. The skewness of PFP decreases stage by stage from 1.0 to 1.5 with the development of cervical diseases; (2) the mean and mode values of PFP both rise from 40.5 to 42.5 as cervical lesion progresses.

Visually, in this three-dimensional coordinate system composed of typical statistical features, cervical precancerous samples at adjacent pathological stages can be separated along the $N$ direction. To quantitatively verify the CIN discrimination ability of PFP's statistical characteristics, we mapped the threedimensional characteristic values of the 60 validation samples to the line $N$. As shown in Fig. 6 (c), the cervical precancer samples are long the $x$-axis, and the $y$-axis represents the values of projections of three statistical features on $N$. We adopted the one-sided t-test of hypothesis testing to assess the probability of the null hypothesis. It shows that the PFP's statistical characteristics are statistically different at different cervical precancer stages ( $\mathrm{P}$-values are all near to 0 between adjacent stages). Therefore, the PFP's statistical parameters are closely related to the microstructural variations as cervical lesion progresses, which can be physically explained to an extent and considered as indicators for quantitative CIN classification. 

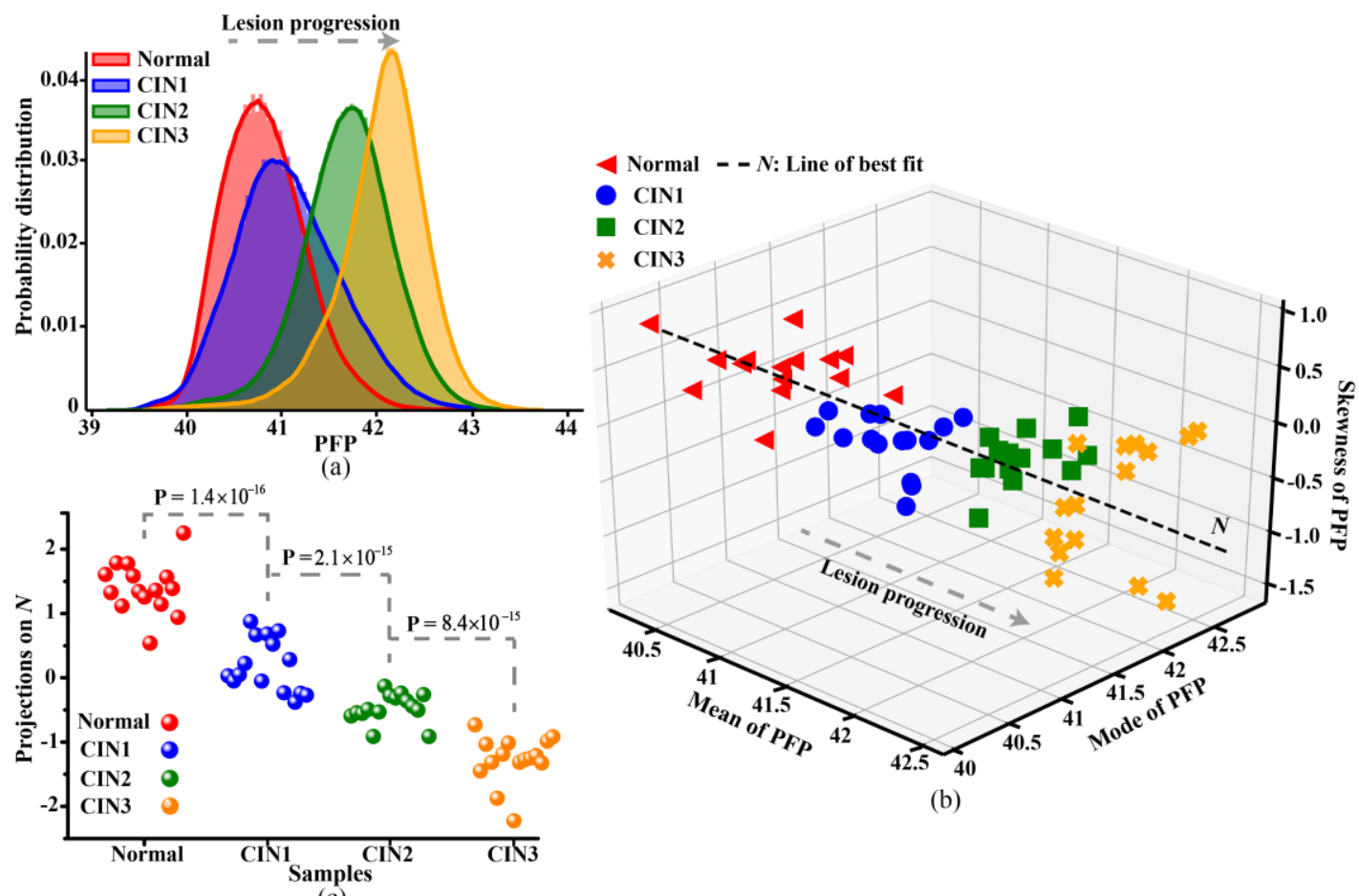

(b)

(c)

Fig. 6. Statistical analysis of the derived PFP in the test samples. (a) PFP's probability distribution of different cervical tissue samples - Normal (red line), CIN1 (blue line), CIN2 (green line), and CIN3 (orange line). The areas under the probability curves are normalized to 1, and the horizontal axis is divided into 50000 parts. (b) PFP's three typical statistical features (mean, mode, and skewness) in the test samples - Normal (red triangle), CIN1 (blue circle), CIN2 (green square), and CIN3 (orange cross). $N$ is the line of best fit obtained using PCA's first component, which can be used for describing the features' variations in the three-dimensional coordinate system. (c) Projections on $N$ of three typical statistical features of cervical tissue samples - Normal (red solid circle), CIN1 (blue solid circle), CIN2 (green solid circle), and CIN3 (orange solid circle). P is the P-value between the two sets of experimental data in two adjacent pathological stages, which is obtained by the t-test $(\mathrm{P}<0.05$ is significant).

\section{E. PFP Performance for CIN Classification}

In order to make this method more practical in the clinic, a CIN classifier - including a multi-class SVM to directly predict the four pathological stages and a binary-class SVM for screening of abnormal samples from normal samples - was trained using the 60 validation samples (See Methods. D. 4)). After obtaining an optimized four-class SVM classifier and an optimized binary-class SVM classifier by 5-fold crossvalidation, we tested their performance on a test set of 40 patients (10 cases from each pathological stage, which did not overlap with samples in the training and validation sets). The 40 test samples' 8 statistical features were fed into the classifier, and various performance measures - accuracy, precision, recall, F1-Score, and area under ROC curve (AUC) - were calculated to analyze the classification results. We can observe from Table I that the accuracy on the classification of Normal vs CIN is 95.0\%. The sensitivity (recall) of PFP in screening cervical lesion tissue samples from the test samples can achieve $96.7 \%$. Considering the color and the Mueller matrix images of H\&E pathological slides were obtained under a low magnification objective lens, the PFP with high sensitivity for CIN diagnosis has potential for rapid and quantitative screening of the abnormal precancerous epithelium regions from the normal epithelium regions.
Furthermore, to evaluate PFP's performance on the classification of CIN at four pathological stages, we calculated the four-class classifier's weighted average performance [49], in which accuracy, precision, and recall achieve $90.0 \%, 92.0 \%$, and $89.9 \%$, respectively. For CIN grading requiring fine diagnosis, the PFP's precision can achieve $92.0 \%$. Therefore, the PFP derived by the statistical distance-based ML classifier has high sensitivity for screening of cervical lesion pathological tissues from the test samples and has high precision in the grading of cervical lesions at adjacent stages.

TABLE I

Cin Classification Results on TeSt SAMPLES

\begin{tabular}{llllll}
\hline \hline $\begin{array}{l}\text { Scoring } \\
\text { Scheme }\end{array}$ & Accuracy & Precision & Recall & $\begin{array}{r}\text { F1- } \\
\text { Score }\end{array}$ & AUC \\
\hline \multicolumn{7}{c}{ The two-class SVM classifier } \\
\hline $\begin{array}{l}\text { Normal } \\
\text { vs CIN }\end{array}$ & 0.950 & 0.917 & 0.967 & 0.937 & 0.998 \\
\hline \multicolumn{5}{c}{ The four-class SVM classifier } \\
\hline $\begin{array}{l}\text { Normal } \\
\text { vs CIN1 }\end{array}$ & 0.900 & 0.920 & 0.899 & 0.898 & 0.971 \\
vs CIN2 \\
vs CIN3
\end{tabular}




\section{DISCUSSION}

In this study, we proposed a dual-modality ML framework combining polarization imaging and traditional microscopy imaging, showing its practicable prospect for accurate and quantitative classification of CIN. By taking advantages of a Unet architecture for image segmentation, we trained a statistical distance-based ML classifier to derive a PFP as a linear combination of a small number of PBPs, related with specific microstructural variations with lesion progression, enabling computer aided diagnosis of Normal, CIN1, CIN2, and CIN3.

The first step is the segmentation of epithelium regions to facilitate extraction of lesion features and assist CIN classification. There have been various techniques to segment epithelial regions in cervical digital H\&E images with the help of deep learning techniques. In this study, we adopted a U-net architecture for epithelium segmentation in digitized cervical H\&E images. The model was evaluated on digitized histology epithelial images from 40 patients. The results indicate that this method yields precise segmentations of epithelium region, and the segmentation achieves the accuracy of $94.4 \%$ and the Dice score of $90.1 \%$, providing reliable epithelial maps for CIN quantitative diagnosis based on the sample polarization characteristics.

After segmenting the epithelium regions by U-net and mapping the segmentation mask onto the corresponding cervical sample's PBPs to select the target pixels as input data, the statistical distance-based ML classifier works and yields precise CIN classification. Here, we designed a loss function according to the requirements of cervical pathological diagnosis in clinical practice and data characteristics in polarimetry. By maximizing the energy distance between polarimetry feature distributions of Normal and CIN1 pathological tissues, the loss function decreases gradually until converging to optimal solutions and outputs the PFP as a simplified linear combination of PBPs. Then we calculated and analyzed the PFP's statistical features of 60 cervical precancerous samples at different pathological stages. It is verified that the statistical behavior of PFP values is very similar to the distribution of immature atypical cells across the epithelium region as the severity of the dysplasia increases.

We evaluated the performance of statistical parameters of PFP on CIN classification by a four-class linear SVM and a binary linear SVM classifier, and the performance measures show that (1) for cervical lesion screening requiring high sensitivity: the accuracy of PFP on the classification of Normal vs CIN is $95.0 \%$, and the corresponding sensitivity is $96.7 \%$, indicating the PFP has high sensitivity in the screening of cervical lesion pathological tissues, and (2) for CIN grading requiring fine diagnosis: the PFP's weighted average accuracy and precision on the four classification can achieve $90.0 \%$ and $92.0 \%$ respectively, meaning that PFP has high precision in the grading of cervical lesions at neighboring stages. Therefore, the polarization-imaging-based ML framework has potential to serve as a powerful tool in segmenting the epithelial regions and provide diagnosis indicators for quantitatively classifying cervical pathological tissues, which is expected to automate the diagnosis process and reduce the workload of pathologists.

Compared with routine computational digital pathology methods under standard microscopes, the proposed approach has some unique advantages: (1) We design the loss function for the ML classifier based on statistical distance to derive the PFP as the diagnostic indicator according to the polarimetry data characteristics and clinical application requirements. The linearity and simplicity of this model make it more general and have less requirements for training samples. (2) Unlike complex deep-learning-based model for CIN classification, the derived PFP is a simplified linear function of the PBPs with physical meanings and has close relationship with the variation of target pathological feature, providing quantitative indicators for characterizing lesion progression and allowing in-depth analysis of physical interpretation. (3) Mueller matrix images and $H \& E$ images of the whole slide were obtained by a low cost and fast imaging Mueller matrix microscope under a low magnification objective. The PFP with high sensitivity has potential to rapidly scan and quantitatively analyze the whole pathological slide in a low-resolution and wide-field system, showing good prospect for cancer primary screening in clinical practice. (4) The dual-modality framework takes advantages of the samples' macro-structure information obtained by microscopy imaging technology and micro-structure information extracted by polarimetry method for assisting accurate and quantitative diagnosis, bringing new possibilities for current image-heavy digital pathology.

\section{ACKNOWLEDGMENT}

We thank Zhi Wang and Ruqi Huang for helpful feedback on the manuscript.

\section{REFERENCES}

[1] K. Bera, K. A. Schalper, D. L. Rimm, V. Velcheti, and A. Madabhushi, "Artificial intelligence in digital pathology-new tools for diagnosis and precision oncology," Nat. Rev. Clin. Oncol., vol. 16, no.11, pp. 703-715, Nov. 2019.

[2] Y. Rivenson et al., "Virtual histological staining of unlabelled tissueautofluorescence images via deep learning," Nat. BioMed. Eng., vol. 3, pp. 466-477, Jun. 2019.

[3] K.-H. Yu et al., "Predicting non-small cell lung cancer prognosis by fully automated microscopic pathology image features," Nat. Commun., vol. 7, Aug. 2016, Art. no. 12474.

[4] N. Coudray et al., "Classification and mutation prediction from non-small cell lung cancer histopathology images using deep learning," Nat. Med., vol. 24, no. 10, pp. 1559-1567, Sep. 2018.

[5] F. Bray, I. Soerjomataram, R. L. Siegel, L. A. Torre, and A. Jemal, "Global cancer statistics 2018: GLOBOCAN estimates of incidence and mortality worldwide for 36 cancers in 185 countries," CA Cancer J. Clin., vol. 68 , no. 6 , pp. $394-424$, Nov. 2018.

[6] M. Arbyn, E. Weiderpass, L. Bruni, S. Silvia, and F. Bray, "Estimates of incidence and mortality of cervical cancer in 2018: a worldwide analysis," Lancet Glob Health, vol. 8, no. 2, pp. 191-203, Dec. 2019.

[7] S. E. Waggoner, "Cervical cancer," Lancet, vol. 361, no. 9376, pp. 22172225, Jun. 2003.

[8] L. Denny and W. Prendiville, "Cancer of the cervix: Early detection and cost-effective solutions," Int. J. Gynaecol. Obstet., vol. 131, no. Suppl. 1, pp. S28-S32, Oct. 2015.

[9] B. A. Erickson, A. B. Olawaiye, A. Bermudez, and E. C. Grendys, "Cervix uteri," in AJCC Cancer Staging Manual. Berlin Heidelberg, Springer, 2017, pp. 649-659. 
[10] L. H. Ellenson and E. C. Pirog, "The female genital tract," in Robbins and Cotran Pathologic Basis of Disease., Philadelphia, Elsevier Saunders, 2014, pp. 1408-1466.

[11] L. He, L. R. Long, S. Antani, and G. R. Thoma, "Computer assisted diagnosis in histopathology," in Sequence and Genome Analysis: Methods and Applications. Hongkong, iConcept, 2011, pp. 271-287.

[12] Q. Zhang, L. Kuhn, L. A. Denny, M. D. Souza, and T. C. Wright, "Impact of utilizing p16ink4a immunohistochemistry on estimated performance of three cervical cancer screening tests," Int. J. Cancer, vol. 120, no. 2, pp. 351-356, Jan. 2007.

[13] S. De et al., "A fusion-based approach for uterine cervical cancer histology image classification," Comput. Med. Imaging Graph., vol. 37, no. 7-8, pp. 475-487, Oct. 2013.

[14] Y. Wang, D. Crookes, O. S. Eldin, S. Wang, P. Hamilton, and J. Diamond, "Assisted diagnosis of Cervical Intraepithelial Neoplasia (CIN)," IEEE J. Sel. Top. Signal Process, vol. 3, no. 1, pp. 112-121, Feb. 2009.

[15] S. Sornapudi et al., "EpithNet: Deep regression for epithelium segmentation in cervical histology images," J. Pathol. Inform., vol. 11, pp. 1-10, Mar. 2020.

[16] S. Sornapudi et al., "Cervical whole slide histology image analysis toolbox," medRxiv, early access, Jul. 2020, doi: 10.1101/2020.07.22.20160366.

[17] J. Xu, X. Luo, G. Wang, H. Gilmore, and A. Madabhushi, "A Deep Convolutional Neural Network for segmenting and classifying epithelial and stromal regions in histopathological images," Neurocomputing, vol. 191, pp. 214-223, May. 2016.

[18] O. Ronneberger, P. Fischer, and T. Brox, "U-Net: Convolutional networks for biomedical image segmentation," 2015, arXiv:1505.04597. [Online]. Available: $\underline{\text { https://arxiv.org/abs/1505.04597 }}$

[19] F. Isensee, P. Jaeger, S. Kohl, J. Petersen, and K. Maier-Hein, "nnU-Net: a self-configuring method for deep learning-based biomedical image segmentation," Nat. Methods, vol. 18, no. 2, pp. 203-211, Feb. 2021.

[20] W. Bulten, P. Bandi, J. Hoven, R. Vandeloo, and G. Litjens, "Epithelium segmentation using deep learning in H\&E-stained prostate specimens with immunohistochemistry as reference standard," Sci. Rep., vol. 9, no. 1, Jan. 2019, Art. no. 864.

[21] N. Ghosh and I. A. Vitkin, "Tissue polarimetry: concepts, challenges, applications, and outlook," J. Biomed. Opt., vol. 16, no. 11, p. 110801, Nov. 2011.

[22] S. Alali and I. A. Vitkin, "Polarized light imaging in biomedicine: Emerging Mueller matrix methodologies for bulk tissue assessment," $J$. Biomed. Opt., vol. 20, no. 6, p. 061104, Mar. 2015.

[23] J. Qi and D. S. Elson, "Mueller polarimetric imaging for surgical and diagnostic applications: A review," J. Biophotonics, vol. 10, no. 8, pp. 950-982, Aug. 2017.

[24] P. Schucht et al., "Visualization of white matter fiber tracts of brain tissue sections with wide-field imaging Mueller polarimetry," IEEE T. Med. Imag., vol. 39, no. 12, pp. 4376-4382, Nov. 2020.

[25] J. Qi and D. S. Elson, "A high definition Mueller polarimetric endoscope for tissue characterization," Sci. Rep., vol. 6, May. 2016, Art. no. 25953.

[26] Y. Dong, H. He, W. Sheng, J. Wu, and H. Ma, "A quantitative and noncontact technique to characterize microstructural variations of skin tissues during photo-damaging process based on Mueller matrix polarimetry," Sci. Rep., vol. 7, Oct. 2017, Art. No. 14702.

[27] S. Y. Lu and R. A. Chipman, "Interpretation of Mueller matrices based on polar decomposition," J. Opt. Soc. Am., vol. 13, no. 5, pp. 1106-1113, May. 1996.

[28] H. He et al., "Emerging New Tool for characterizing the microstructural feature of complex biological specimen," J. Lightwave Technol, vol. 37, no. 11, pp. 2534-2548, Sep. 2019.

[29] H. He et al., "A possible quantitative Mueller matrix transformation technique for anisotropic scattering media," Photon. Lasers Med., vol. 2, no. 2, pp. 129-137, Feb. 2013.

[30] P. Li, D. Lv, H. He, and H. Ma, "Separating azimuthal orientation dependence in polarization measurements of anisotropic media," Opt. Express, vol. 26, no. 4, pp. 3791-3800, Feb. 2018.

[31] Y. Dong et al., "Quantitatively characterizing the microstructural features of breast ductal carcinoma tissues in different progression stages by Mueller matrix microscope," Biomed. Opt. Express, vol. 8, no. 8, pp. 3643-3655, Jul. 2017.

[32] T. Liu et al., "Distinguishing structural features between Crohn's disease and gastrointestinal luminal tuberculosis using Mueller matrix derived parameters," J. Biophotonics, vol. 12, no. 12, p. e201900151, Aug. 2019.
[33] Y. Wang et al., "Differentiating characteristic microstructural features of cancerous tissues using Mueller matrix microscope," Micron, vol. 79, pp. 8-15, Dec. 2015.

[34] Y. Wang et al., "Mueller matrix microscope: a quantitative tool to facilitate detections and fibrosis scorings of liver cirrhosis and cancer tissues," J. Biomed. Opt., vol. 21, no. 7, p. 071112, Jul. 2016.

[35] Y. Dong et al., "Deriving polarimetry feature parameters to characterize microstructural features in histological sections of breast tissues," IEEE T. Bio-Med. Eng., vol. 68, no. 3, pp. 881-892, Jan. 2021.

[36] E. Du et al., "Mueller matrix polarimetry for differentiating characteristic features of cancerous tissues," J. Biomed. Opt., vol. 19, no. 7, p. 076013, Jul. 2014

[37] Y. Dong et al., "Characterizing the effects of washing by different detergents on the microstructures of silk fibers using Mueller matrix polarimetry," Int. J. Mol. Sci., vol. 17, no. 8, pp. 1301-1315, Aug. 2016.

[38] Y. Shen et al., "Comparative study of the influence of imaging resolution on linear retardance parameters derived from the Mueller matrix," Biomed. Opt. Express, vol. 12, no. 1, pp. 211-225, Dec. 2020.

[39] D. H. Goldstein, "Mueller matrix dual-rotating retarder polarimeter," Appl. Opt., vol. 31, no. 31, pp. 6676-6683, Nov. 1992.

[40] P. Ghassmi, L. T. Moffatt, J. W. Shupp, and J. C. Ramella-Roman, “A new approach for optical assessment of directional anisotropy in turbid media," J. Biophotonics, vol. 9, no. 1-2, pp. 100-108, Jan. 2015.

[41] M. Sun et al., "Probing microstructural information of anisotropic scattering media using rotation-independent polarization parameters," Appl. Opt., vol. 53, no. 14, pp. 2949-2955, 2014.

[42] E. GarciaCaurel et al., "Advanced Mueller ellipsometry instrumentation and data analysis," in Ellipsometry at the Nanoscale. Berlin Heidelberg, Springer, 2013, pp. 31-143.

[43] R. Shekhar and V. Zagrodsky, "Mutual information-based rigid and nonrigid registration of ultrasound volumes," IEEE Trans. Med. Imag., vol. 21, no.1, pp. 9-22, Jan. 2002.

[44] M. Jenkinson and S. Smith, "A global optimization method for robust affine registration of brain images," Med. Image Anal., vol. 5, no. 2, pp. 143-156, Jun. 2001

[45] M. G. Bellemre et al., "The Cramer distance as a solution to biased Wasserstein gradients," 2017, arXiv: 1705.10743. [Online], Available: http://arxiv.org/abs/1705.10743

[46] L. Armi and S. F. Ershad, "Texture image analysis and texture classification methods - A review," Int. J. Pattern. Recogn., vol. 2, no. 1, pp. 1-29, Jan. 2019

[47] C. Cortes and V. Vapnik, "Support-vector networks," Mach. Learn., vol. 20, no. 3, pp. 273-297, Sep. 1995.

[48] B. Moore, "Principal component analysis in linear systems: Controllability, observability, and model reduction," IEEE T. Automat. Contr., vol. 26, no. 1, pp. 17-32, Mar. 1981.

[49] M. Grandini, E. Bagli, and G. Visani, "Metrics for multi-class classification: an overview," 2020, arXiv: 2008.05756v1. [Online], Available: http://arxiv.org/abs/2008.05756v1 